\title{
The Empirical Analysis of the System of Risk Management on Small and Medium-Size Enterprises
}

\author{
Raigul Doszhan ${ }^{1}$, Akmaral Mustafina $^{1} \&$ Gulnazym Supugalieva ${ }^{1}$ \\ ${ }^{1}$ Al-Farabi Kazakh National Univeristy, Almaty, Kazakhstan \\ Correspondence: Raigul Doszhan, Al-Farabi Kazakh National Univeristy, Almaty, Kazakhstan. E-mail: \\ info@kaznu.kz
}

Received: April 23, 2015 Accepted: May 5, 2015 Online Published: July 6, 2015

doi:10.5539/ass.v1 $1 \mathrm{n} 21 \mathrm{p} 240$

URL: http://dx.doi.org/10.5539/ass.v11n21p240

\begin{abstract}
Risk management is an important element among the activities of small and medium-sized enterprises. It is necessary for SMEs to be able to effectively manage risks to survive in highly competitive conditions. Although there are positive dynamics of growth in the number of SMEs in Kazakhstan, statistics show unprofitability in their activities. The article shows an empirical method of analysis where 82 representatives of small and medium-size enterprises where interviewed. This article addresses the problems of SMEs in the sphere of trade, manufacturing, construction and services, which have been studied by the author in recent years and considers the role of external consultants in the risk management activities of SMEs.
\end{abstract}

Keywords: small and medium enterprises, risks problems of small and medium businesses, Cronbach index

\section{Introduction}

An empirical knowledge is a complex of statements about real empirical objects. The empirical knowledge is based on knowledge of acquaintance. A rational factor and its forms (understandings, comprehensions, etc) are present here but have a subordinate value. That's why an analyzed object is reflected primarily from the side of its external links and performances accessible for contemplation and expressing the internal relations (Bryman, 2011).

\section{Method}

The main target of questionnaire method of this article is:

- to find out the entrepreneurs' attitude to risks;

- a condition of risk management on practice of small and medium business;

- an elimination of factors which influence on the system of risk management implementation on the enterprises of small and medium business;

- an elimination of factors which stimulate the system of risk management implementation on the enterprises of small and medium business.

The results received after an interviewing suffered the regression analysis, factor analysis and descriptive analysis.

In total there were 82 enterprises surveyed among which 44 answers were received by means of SurveyMonkey questionnaire and the rest 38 - paper based.

During a survey all the ethical norms were observed. All the survey members were consulted about the purposes and tasks of enquiry. Either a participation in an enquiry was exceptionally voluntary.

The basic instrument of investigation was a questionnaire which consisted of 36 questions. A survey was made on two languages - Russian and Kazakh, but the most entrepreneurs desired to answer in Russian.

From an informative point of view all the questions of questionnaire could be separated minimum on two groups:

1) The first ten questions give an indication about the general characteristics of surveyed objects (size, age of business, legal organizational form, kind of activity, annual turnover) and respondents (sex, age, position, education). 
2) The rest questions of questionnaire (from 11 to 36) are directed on the peculiarities of external and internal business environment, presence or absence of the risk management elements, sufficiency and availability of resources for risks control on enterprise.

From a statistic point of view the questions could be separated on three groups:

1) The questions in which a respondent should choose one of offered answers (questions from 2 to 11 and also $18,20,23,25,27,28,30,33,34$ and 36). To each of these questions in the limits of our statistical analysis a corresponding variable is set $\mathrm{q} j$, where $j$ is equal to the number of question in questionnaire which is categorical (nominal) and takes the integer values from 1 to $n$, where $n$ is the quantity of variants of answers on this question. Thus each variant of answer corresponds to its own variate value.

2) The questions which stipulated an opportunity of simultaneous choice of several variants of answer (questions from 12 to 17, 19, 21, 22, 24, 29, 31, 32 and 35). In this case to each variant the other variable corresponded $-\mathrm{q} j \_k$, where $j$ is equal to the number of question in questionnaire and $k-$ to an order number of the answer's variant for this question. These variables are binary (also correspond to the number of nominal variables), i.e. take a value 1 for those respondents who chose this variant and 0 - if otherwise.

3) The question 26 in which the respondents were asked to determine a level of significance of different risks for business in numbers. For each risk a variable q26_k was created where $k$ is a numerical order which corresponds to set type of risk in the list of all risks of this question. This variable is serial, i.e. allows ranging the risks according to their level of significance for the entities of small business.

\section{Results}

There were 82 respondents who took participation in an investigation. Their answers on basic questions are shown in the Figures 1-9.

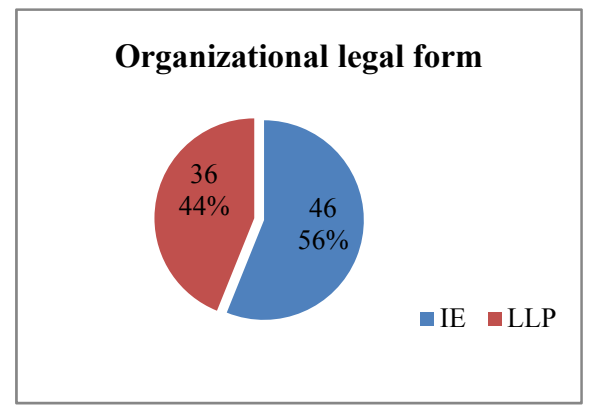

Figure 1.

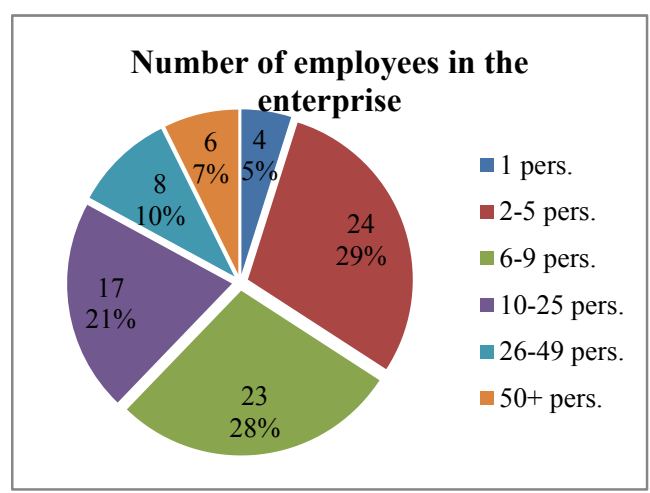

Figure 3.

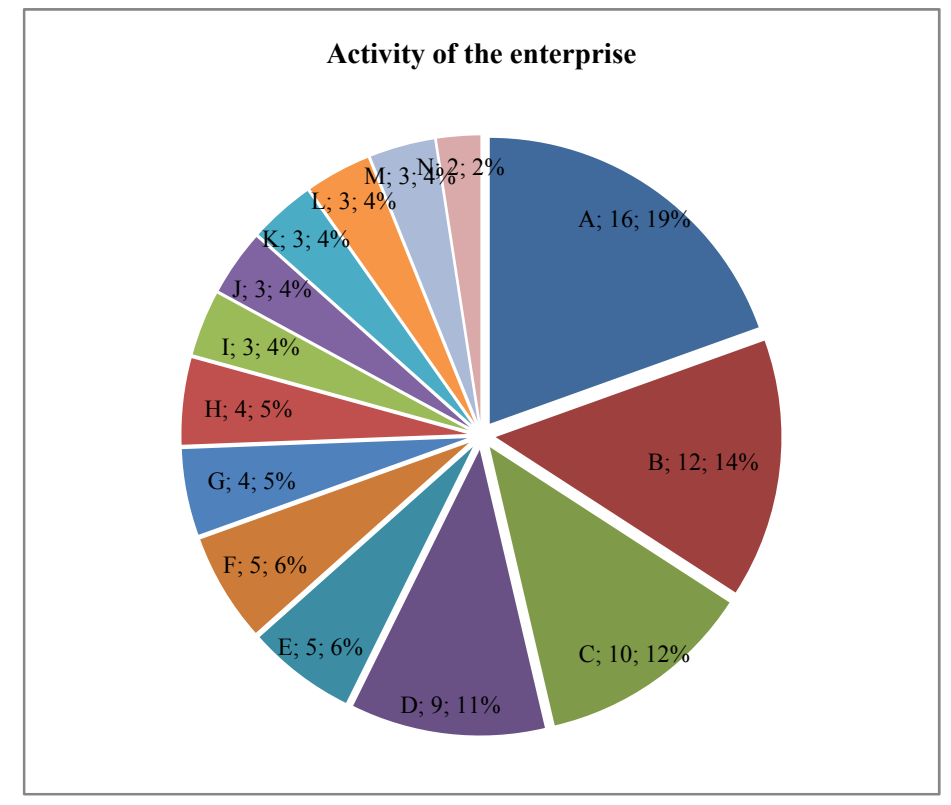

Figure 2 .
Services for accommodation and meals $\mathrm{H}$

Professional, scientific and technical activities I

Other services $\mathrm{J}$

trade $\mathrm{K}$

Health and social services L

production $\mathrm{M}$

Administrative and support services $\mathrm{N}$ other

Transportation and warehousing

Information and communication

Financial and insurance activities

Education

Arts, entertainment, recreation Real estate transactions 


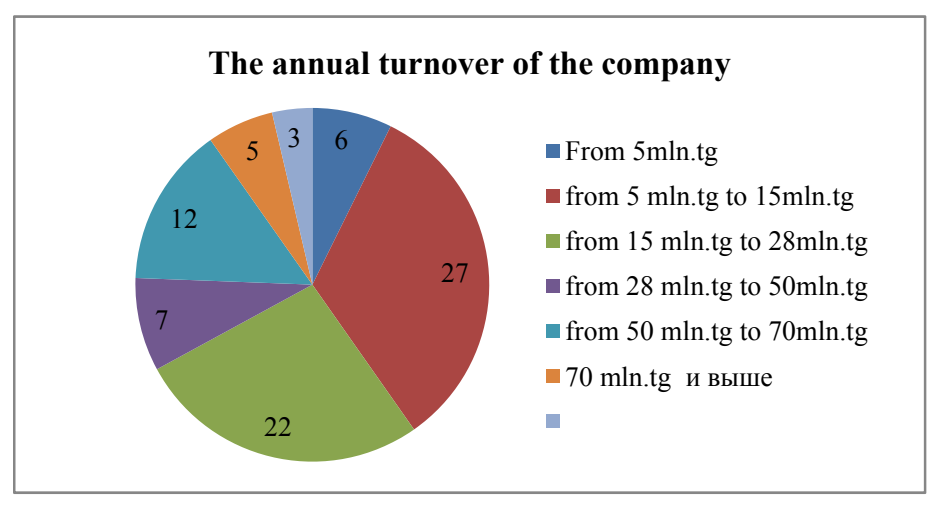

Figure 4.

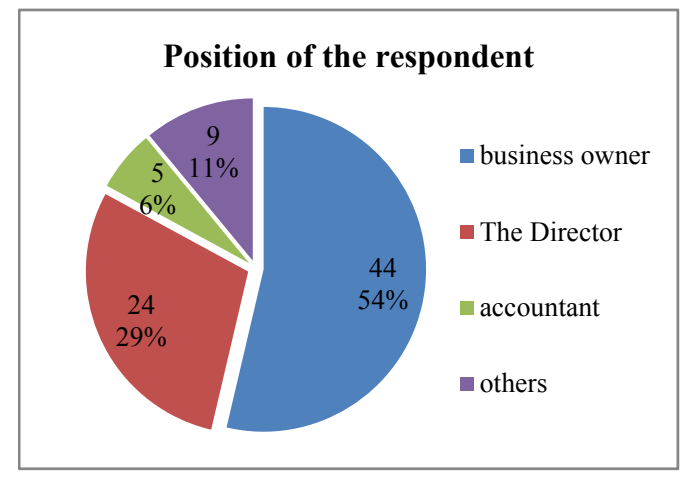

Figure 6.

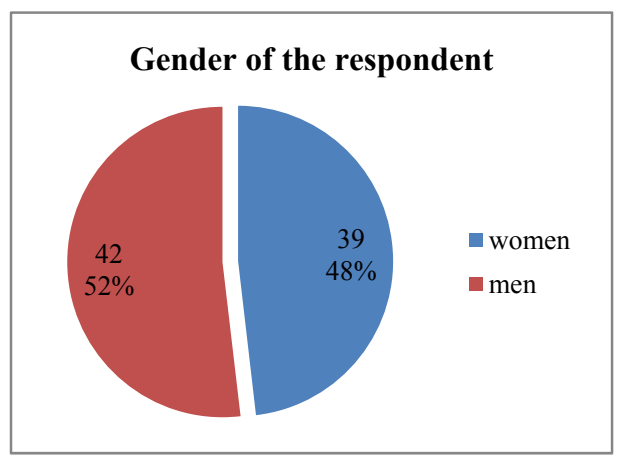

Figure 8.

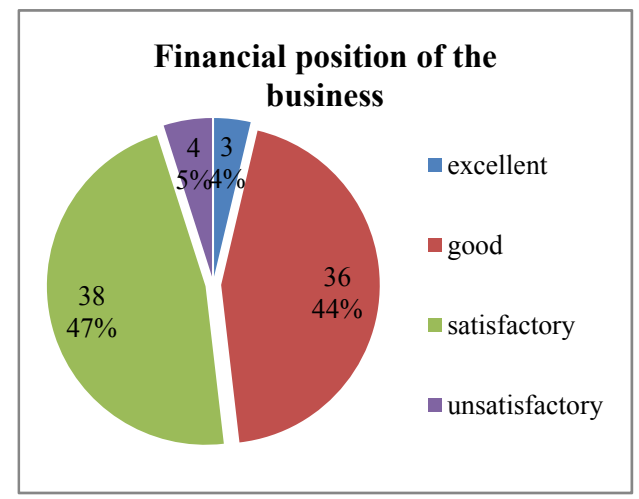

Figure 5.

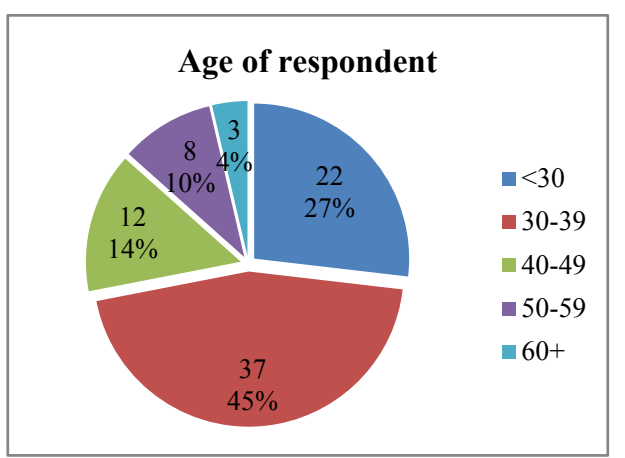

Figure 7.

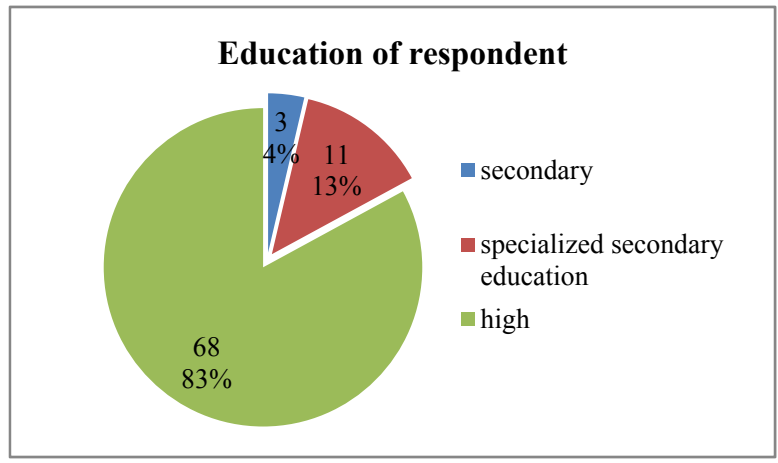

Figure 9.

In whole an under-representation of business entities with unsatisfied financial condition including determined by objective standards is a common practice (and problem) of investigations because usually such entities refuse to take part in enquiries. Consequently we cannot in the limits of our investigation determine how the presence of risk management system can assist to overcome the financial difficulties of business which is let's say on the verge of bankruptcy, i.e. our results relate first of all to stably functioning small enterprises.

The last suggestion is supported also by the fact that the respondents' anticipations regarding the perspectives of the national economy development are quite positive: $70 \%$ of them wait its growth (stable and inconspicuous), $12 \%$ consider that an economic dynamics will stay unchanged and only $16 \%$ and $1 \%$ accordingly predict an inconspicuous or strong fall of the Kazakhstani economy. Moreover basically a subjective assessment of financial condition correlates to these anticipations: the business representatives with unsatisfied financial condition mainly expect fall or stability, with good or satisfied - inconspecious growth or stability, with exelent - only significant growth. It also can proove that a meaning of respondent about a business financial condition depends on his grade of business optimism.

From other side $44 \%$ of surveyed consider that if an economic situation in the country will be unfavourable it will prevent their business development in current year and only $29 \%$ relate a favourable economic situation to the factors of growth. That is no matter what they expact but a small enterpreneurship is quite dependable from 
macroeconomic shocks and this dependance is quite peculiar: if the economy falls it brings the significant losses including in the form of liquidation of enterprises, but if it grows it doesn't always act as a fillip of small business development, because the profits of economic growth are redistributed first of all in favour of big and partially middle companies. In fact 2013 was quite difficult year for the most of surveyed enterprises: a half of them faced with price rise on market, $37 \%$ - with compitition strengthening, $18 \%$ - with lack of cash, $15 \%$ with sales decrease, and $15 \%$ noted that the conditions turned out to be more difficult then they expacted. Only $11 \%$ of all surveyed announced that their business showed out new opportunities and only $6 \%$ of firms increased their sales. The majority (58\%) connects it directly to devaluation. In this connection an attitude of mind of surveyed mainly could be considered optimistic if under these conditions they continue to believe in growth of economy or its stable development.

Whereby we can pay our attention to the fact that the entities of small business mainly rely on the internal sources of development which they can manage (first of all increase of sales and improvement of business model), but a standard basically from negative factors of external environment (rise of prices and rent, unfavourable business climate in the country, strengthening of competition). Besides the most significant types of risk management for them are the financial and market, but the significance of risk management and personnel risk in comparison with them on 1-1.5 points (according to 7 point scale) lower.

Let's pay our attention to the fact that the anticipations of respondents regarding the macroeconomic dynamics depend not only on the level of their optimizm - they are also could be considered highly burdened with "the baggage of the past", because the anticipations and the past experience strongly correlate to each other. Thus, the two thirds of respondents who faced in 2013 a price rise expect a negative influence of this factor in current year, but almost a quarter of those who didn't face it consider that in this year they won't suffer the influence of this factor.

In regard to excess develope on the level of selling market or internal environment of organization the other picture is observed: notwithstanding the past negative experience the major part of respondents expect in future an absence of unfavourable influence from such factors, while the overwhelming majority of those who didn't face them in past continue to consider that in future they won't meet them. For example $67 \%$ of those who suffered an aggravation of firms' competition in 2013 and 85\% of those who didn't have such experience in current year don't relate this factor to the row of deterrents. Practically the analogue figures could be observed in relation to the factor of sales decrease.

What did the entities of small business do in order to overcome the unfavourable conditions of development and prevent negative consequences? Basically improved the work with clients (64\%). Besides $38 \%$ reconsidered the current expenses, $25 \%$ improved marketing, $22 \%$ introduced changes in business process. Only $6 \%$ were forced to leave some markets or change suppliers.

In regard to an assistment from outside more then a half of respondents admited that their business needs outside financing ( $44 \%$ - exactly, $13 \%$ - possible). $11 \%$ recognized problems with financing as a deterrent for business development in current year. And if a quarter of enterprises didn't face the problem of attraction of means from outside resources yet, but practically for all the rest it is linked to difficulties (only $5 \%$ of respondents consider that it is easily feasible). Whereby the funds of enterpreneurship's support are not very popular sources of financing: $72 \%$ of enterprises didn't call in aid to them, $5 \%$ didn't know about their existance, $10 \%$ don't relly this system, and 5\% couldn't receive any assistance from them. Only 7\% had a successful experience of appealation to supporting funds and only for two from 82 surveyed enterprises the means of these funds are one of the sources of their business financing. Less than $60 \%$ of surveyed know about the state entrepreneurship support programs. Mainly the small business exists at its sole cost and expense ( $70 \%$ of firms), by means of friends' and relatives' support (12\%), and from outside sources the most popular are the means of banks (21\%).

A picture of respondents' composition also could be important for interpretation of answers and results of investigation. The majority of surveyed $-83 \%$ are the owners of business or the directors of enterprises, what means that we can rely the received from them data and we could be sure that their answers correspond an actual state of things on enterprises and give an adequate representation of risk management practice on them. $6 \%$ of respondents are the accountants, $11 \%$ - the representatives of other job positions (mainly administrators, secretaries) and in this case we can only hope that the represented in their answers "worldview" reflects reality adequately. At least almost all of them have a high education (12 from 14 people).

At last let's go into the questions which directly relate to risk management in small business.

From 82 enterprises the complete system of risk management works only for 6 (it is $7 \%$ from overall volume of selection), partially - for $19(23 \%)$. A quarter is going to introduce it in near future and by $22 \%$ falls on those 
who still study the base of risk management and think over its advantages for their own business and on those who are not going to introduce such system at all. Whereby an overwhelming majority of surveyed (92\%) would like to manage, assess and control risks of their business better including 16 from 18 who are not going to introduce the system of risk management on their enterprises. Along with that more than a half of such interested (55\%) in their own opinion don't have enough knowledges in the sphere of risk management. The lack of knowledges is observed even where the risks are managed already on a systematic basis: it is actual for $17 \%$ of enterprises with the full system of risk management and for $47 \%$ of firms with partial. The respondents themselves consider a qualification absence in this sphere a main obstacle for the system of risk management introduction ( $32 \%$ of surveyed).

Notwithstanding the absence of the system of risk management on the majority of enterprises almost all the respondents argue that they assess the risks in the process of planning ( $53 \%$ always, $44 \%$ some times). However a half of them don't have a well-defined behaviour strategy in case of risk situations occurance (mainly those who don't assess the risks during planning or do it sometimes).

Consequently the respondents have a desire to manage the risks in small business but suffer an evident lack of any knowledge about the systematic risk management and what advantages it can give to a small enterprise. The last can be reflected including in the fact what instruments of risk management are used by firms on a current moment and how the decisions are made in risk situations. Thus, the main instrument of risk management among the surveyed is avoiding of risk dealings (53\%) and the decisions regarding the risks are took by the majoruty in reliance on the past experience (59\%) and on an intuition (21\%). The most "advanced" methods such as business diversification, usage of the results of mathematical calculations and attraction of outside consultants are applied on less then quarter of enterprises.

Before getting started directly to work with data of our investigation it is necessary to understand how reliable a chosen enquiring instrumentarium occurred, how it corresponds to the tasks of our investigation. We set our mind on determination of how the enterprises of small business behave themselves in regard to risks which they face and for this purpose we asked a row of questions. We'd like to receive the most exact and full answers which will characterize an examined occurrence. Thus, speaking mathematical language in the process of data collection we intend for each observed object $(i)$ to minimize the difference between an effective value for each variable $\left(Q_{i}^{\text {real }}\right)$ and observed $\left(Q_{i}^{\text {obs }}\right)$.

A deviation of one from another refers to the measurement errors of this variable $(M E Q)$ :

$$
Q_{i}^{o b s}=Q_{i}^{\text {real }}+M E Q_{i}
$$

The measurement errors could be casual $\left(M E Q^{\text {rand }}\right)$ and systematic $\left(M E Q^{\text {syst }}\right)$ thus for each observed object $(i)$ :

$$
M E Q_{i}=M E Q^{\text {syst }}+M E Q_{i}^{\text {rand }}
$$

The casual errors always present in the process of measurement, they vary from one observed object to another and could be caused by the unanticipated deviations in understanding and interpretation of questions by separate respondents, by unpredictable difference in the conditions of questionnaire conduction with different participants, etc. It is important to emphasize that the casualty of deviations means not only that they are not constant for all the observed objects but also that their appearance is not connected to any features of these objects. For example an understanding of question is not an answer on it but exactly understanding! And systematically it doesn't depend on sex, age, education, position and other characteristics of respondents.

A mean value of casual errors of measurement for each variable in selection is equal to zero:

$$
\frac{\overline{M E Q^{\text {rand }}}}{=}=\frac{\sum_{1}^{N} M E Q_{i}^{\text {rand }}}{N}=0
$$

The systematic errors of measurement if they occur are present in all the investigations. Their reason is in an incorrectness of measuring instrument (in our case of questionnaire, thus in choice of quantity of questions and variants of answers on them, in descriptions, explanations and requirements to filling the data sheet). If such errors are present thus they are constant for all the selection or proportional to an actual value of variable. In the first case: 


$$
\frac{\overline{M E Q^{s y s t}}}{=} \frac{\sum_{1}^{N} M E Q^{\text {syst }}}{N}=M E Q^{\text {syst }} \neq 0
$$

In the second case:

$$
\overline{M E Q^{\text {syst }}}=\frac{\sum_{1}^{N} a^{*} Q_{i}^{\text {real }}}{N}=a \overline{Q^{\text {real }}} \neq\left\{Q^{\text {real }} \neq 0\right\} \neq 0
$$

where $a$ is a factor of proportionality and $a>0$.

In the process of statistical data analysis we transfer to the usage of mean values of variables $\left(\overline{Q^{\text {obs }}}\right)$ and characterize a corresponding criterion also in the mean $\left(Q^{\text {real }}\right)$ where:

$$
\overline{Q^{o b s}}=\frac{\sum_{1}^{N} Q_{i}^{o b s}}{N} \overline{Q^{\text {real }}}=\frac{\sum_{1}^{N} Q_{i}^{\text {real }}}{N}
$$

$N$ is a quantity of observances (of respondents).

In order we could talk about an adequacy of received data it is necessary that $\overline{Q^{\text {obs }}}=\overline{Q^{\text {real }}}$.

If there are the measurement errors we have:

Where

$$
\begin{gathered}
\overline{Q^{\text {obs }}}=\overline{Q^{\text {real }}}+\overline{M E Q} \\
\overline{M E Q}=\overline{M E Q^{\text {rand }}}+\overline{M E Q^{s y s t}}
\end{gathered}
$$

Considering the features of casual and systematic errors if the last are absent, thus:

$$
\overline{Q^{\text {obs }}}=\overline{Q^{\text {real }}}+\overline{M E Q}=\overline{Q^{\text {real }}}+\overline{M E Q^{\text {syst }}}=\left\{\text { ecли } \overline{M E Q^{\text {syst }}}=0\right\}=\overline{Q^{\text {real }}}
$$

otherwise:

$$
\overline{Q^{\text {obs }}}=\overline{Q^{\text {real }}}+\overline{M E Q} \neq\left\{\text { eсли_ } \overline{M E Q^{s y s t}} \neq 0\right\} \neq \overline{Q^{\text {real }}}
$$

Consequently the presence of casual errors doesn't prevent the usage of statistical analysis, i.e. doesn't distort the results and allows considering them adequate for all the selection. As to the systematic errors if observer knows about their presence, reasons and nature (constant or proportional), thus in order to receive more adequate results he usually can insert the corresponding adjustments in data or evaluations or under the repeated data collection to change the applied instrumentarium and its organization. Otherwise an understanding of analyzed phenomenon based on observed data will give to an investigator an inadequate picture of reality.

In this work in order to prove that received answers on a variety of questions of our questionnaire adequately determine one general investigated problem sphere we check them on an internal conformity by means of factor $\alpha$ (alpha) of Cronbach. This test is built on evaluation of correlation between the dispersion of actual values and the dispersion of observed values of variables. The dispersion of observed values for each variable consists of the dispersion of actual values (answers of respondents) and the dispersion of measurement errors (Cronbach , 1951).

The $\alpha$ of Cronbach is a figure from 0 to 1 which shows how adequately the tested variables determine one phenomenon. It is considered that if $0.7 \leq \alpha \leq 1$ thus the data are internally conformal, the measurement errors are casual, the observed dependences are close to actually existing and the chosen enquiring instrumentarium is reliable (Nunnally, 1978). It should be noted that the high values of the $\alpha$ of Cronbach are not obligatory prove an internal data consistency because could be the result of simple introduction in questionnaire of a large number of questions which measure the same phenomenon but worded differently (Cortina, 1993). That's why to prove an investigating instrumentarium as reliable on the basis of test of the $\alpha$ of Cronbach is possible only in case if the questions in questionnaire are not only combined by a thematic communality but each separate variable in analysis is intended to collect for investigator some unique information which couldn't be received from data of other variables. For this purpose in the indicator values, i.e. in the answers of respondents on the same question or subitem a definite variation should be observed.

It is necessary to emphasize here that the $\alpha$ of Cronbach is estimated exactly by variables but not by the questions of questionnaire (the difference between them has been shown before, in the section 1.1) and it means 
that it can take the high values in case if there are little number of questions in questionnaire but at least one of them has a large number of variants of answers. In order it didn't prevent the evaluation of data consistency dimension it is necessary to make a clear distinction between these variants according to subject matter in a way where they couldn't cross significantly. Accordingly we determine in our data on what number of variables the evaluations were received $(n)$.

By means of the $\alpha$ of Cronbach an index of measurement errors $(v)$ could be estimated either: it is equal to a difference between 1 and a square of the $\alpha$ of Cronbach. If this index is high (i.e. the $\alpha$ of Cronbach is small) it could be connected as a minimum with two reasons. First of all the investigated problem actually could be multi aspect and the answers on different items of questionnaire characterize its different sides and it should be considered in the following analysis. Secondly it can occur that the answers on different groups of questions characterize the completely different phenomena excluding the directly investigated. Thirdly there the systematic measurement errors are present connected to incorrectness of selection methods of investigations (formation of selection), data collection or investigation instrument itself (questionnaire). For example the number of questions in questionnaire was insufficient for an adequate description of interesting for investigator problem or the data contain a lot of omissions (questions left by at least some of respondents without answers) or the questions were worded incorrectly and as a result of which misunderstood by all the respondents. All these are the potential sources of measurement errors.

If the $\alpha$ of Cronbach takes low values, thus unfortunately it is impossible to specify according to only this evaluation by what of the listed before reasons it was caused, as well as if we don't doubt in correctness of the investigation organization and the process of data collection we cannot say what number of potentially possible phenomena are hidden in our data and what are these phenomena.

We evaluated the $\alpha$ of Cronbach according to all the variables which reflect the condition of external and internal business environment and the system of risk management on enterprises (the questions of questionnaire from 11 to 36) excluding those for which a zero or low variation of values were observed (in other words excluding those questions and subitems on which all or almost all of respondents gave the same answers). Their exclusion is necessary because in otherwise we can receive an overestimated value of the $\alpha$ of Cronbach and make an improper conclusion of internal data consistency degree (actually it will be less than evaluated). Because of an analogue reason we additionally estimated the $\alpha$ of Cronbach excluding the questions which cross with each other intensively within the meaning (in our questionnaire there were two pairs of such questions: 14 and 16, 28 and 34; whereby one of the questions were excluded from each pair of evaluation).

Besides we evaluated the $\alpha$ of Cronbach as to all the enterprises of our selection and exceptionally to those the representatives of which answered all the questions (there were 51 of such in our selection). In the first case the evaluation is made according to pair correlations between the variables, in the second - according to general. If there were no missed answers thus the data gave the same results. If the omissions were present but they born an occasional character or their number was relatively not large so it is worth to rely on the results of the second approach. Under the casualty of omissions the fact that they don't depend on the characteristics of respondents and observed phenomenon is understood, i.e. there is no fact that some groups of respondents significantly more often leave some definite questions without answers than others.

There is no special methodology of checking the omissions on casualty, but we evaluated the regressional dependences of missed answers presence from the main features of respondents and their business. The results are represented in the Table 2.1 of annex 2. They imply that there are no significant differences between the separate groups of enterprises according to the presence of missed answers excluding the case of the enterprises' separation according to the presence of the system of risk management: if it is present (full-value or partial proceeding from the answers on the $30^{\text {th }}$ question of questionnaire) thus the respondents significantly more often answered on all the questions of questionnaire than those who noted an absence of such system in their business. In whole it is an expected result because those who don't manage the risks of their business first of all can worse understand some questions of questionnaire and secondly can consider this problematic not actual and uninteresting for themselves. Along with that it is necessary to understand that these observations probably (according to the fact what questions were left without answers) will be excluded from the following analysis including from an evaluation of regression equations in the result of what we can receive an incomplete or incorrect information about the factors of the system of risk management presence on enterprises.

That's why in our case we can consider the values of the $\alpha$ of Cronbach evaluated by means of the first and the second approaches as low and high limits of a true value of the $\alpha$ of Cronbach consequently. All the received evaluations are represented in the Table 1. 
Table 1. The values of the $\alpha$ of Cronbach and an index of measurement errors $v$ ( $n$ is a number of variables included in the analysis)

\begin{tabular}{ccc}
\hline & $\begin{array}{c}\text { All the observations } \\
\text { (respondents) }\end{array}$ & $\begin{array}{c}\text { Without observations with missed values (only } \\
\text { the respondents who answered on all the } \\
\text { questions of questionnaire) }\end{array}$ \\
\hline $\begin{array}{c}\text { All the variables except those with zero } \\
\text { and low variation }(n=105)\end{array}$ & $\alpha=0.74$ & $\alpha=0.86$ \\
$v=0.45$ & $\alpha=0.26$ \\
\hline Without questions 16, 34 (n=98) & $\alpha=0.74$ & $\alpha=0.85$ \\
& $v=0.45$ & $\alpha=0.28$ \\
\hline Without questions 14, 28 (n=97) & $\alpha=0.72$ & $v=0.86$ \\
& $v=0.48$ &
\end{tabular}

Notice. The values of the $\alpha$ of Cronbach are evaluated according to standardized variable values. The standardization of data allows giving answers on all questions according to one scale (with a mean value equal to 0 and a dispersion equal to 1 ) in the case when the scales of answers on separate questions don't coincide between each other.

All these values exceed the limit of 0.7 that's why we can consider the applied questionnaire a reliable instrument of studying a condition of the system of risk management on chosen entities of small business.

\section{References}

Bryman, A., \& Bell, E. (2011). Business Research methods (3rd ed., pp. 10-38). Oxford University Press.

Cortina, J. M. (1993). What is Coefficient Alpha? An Examination of Theory and Applications. Journal of Applied Psychology, 78, 98-104.

Cronbach, L. J. (1951). Coefficient Alpha and the Internal Structure of Tests. Psychometrika, 16(3), 297-334.

Nunnally, J. C. (1978). Psychometric Theory (2nd ed.). New York: McGraw-Hill.

\section{Copyrights}

Copyright for this article is retained by the author(s), with first publication rights granted to the journal.

This is an open-access article distributed under the terms and conditions of the Creative Commons Attribution license (http://creativecommons.org/licenses/by/3.0/). 